Original research

\title{
Update on the role of ultrasound guided radiofrequency ablation for thyroid nodule treatment
}

\author{
Maija Radzina a, Vito Cantisani ${ }^{\mathrm{b}}$, Madara Rauda ${ }^{\mathrm{a}}$, Michael Bachmann Nielsen ${ }^{\mathrm{c}}$, \\ Caroline Ewertsen ${ }^{\mathrm{c}}$, Ferdinando D'Ambrosio ${ }^{\mathrm{d}}$, Peteris Prieditis ${ }^{\mathrm{a}}$, Salvatore Sorrenti ${ }^{\mathrm{e}}$, \\ a Diagnostic Radiology Institute, Paula Stradina Clinical University Hospital, Pilsonu Street 13, LV - 1002, Riga, Latvia \\ ${ }^{\mathrm{b}}$ Department of Radiological Sciences, Policlinico Umberto I, Sapienza University of Rome, 00185, Viale Regina Elena 324, Italy \\ ${ }^{c}$ Department of Radiology, University of Copenhagen, Copenhagen, Denmark \\ d Department of Radiological Sciences, Policlinico Umberto I, Univ. Sapienza, Roma, 00185, Viale Regina Elena 324, Italy \\ e Department of Surgical Sciences, Policlinico Umberto I, University Sapienza, Roma, 00185, Viale Regina Elena 324, Italy
}

\section{H I G H L I G H T S}

- We review the status of radiofrequency ablation treatment of thyroid nodules.

- It has a role in thyroid nodule volume reduction and improve patient symptoms.

- Multicenter studies are warranted to better clarify its clinical utility.

\section{A R T I C L E I N F O}

\section{Article history:}

Received 20 December 2016

Received in revised form

7 February 2017

Accepted 8 February 2017

\section{Keywords:}

Radiofrequency ablation

RFA

Radiofrequency thermal ablation

Thyroid

Thyroid gland

Thyroid nodule

Ultrasound

Ultrasonography

\begin{abstract}
A B S T R A C T
Thyroid nodules can be frequently detected in general population, most of them are benign, however malignant cases are rising in the past decades. Ultrasound (US) is the most common imaging modality to assess thyroid nodular lesions, plan patient work-up and guide minimally invasive treatment. The present review paper has been researched to evaluate the current status of radiofrequency ablation (RFA) treatment in order to evaluate indications, techniques, complications, limitations and outcome assessment in benign solid, partially cystic nodules and recurrent malignant nodules. RFA comparison with other ablation techniques has been provided as well. US guided Radiofrequency ablation has been proved to be effective and safe in cases of benign thyroid nodules and a promising treatment method alternative to surgery for patients with recurrent papillary thyroid cancer. It's major role lies in significant reduction of thyroid nodule mean volume and improvement of the patient symptoms. However, future multicenter studies are warranted to better clarify the multi-parametric patient selection criteria and evaluate RFA technique efficacy, advantages and limitations in the variety of thyroid nodular disease.
\end{abstract}

(C) 2017 IJS Publishing Group Ltd. Published by Elsevier Ltd. All rights reserved.

\section{Introduction}

Thyroid nodules are very frequent in the general population, with a prevalence of $20-67 \%$ and of about $50 \%$ in the individuals

\footnotetext{
* Corresponding author. Department of Surgical Sciences, Policlinico Umberto I, Sapienza University of Rome, 00185, Viale Regina Elena 324, Italy.

E-mail addresses: mradzina@gmail.com (M. Radzina),vito.cantisani@uniroma1. it (V. Cantisani), madara.rauda@gmail.com (M. Rauda), mbn@dadlnet.dk (M.B. Nielsen), caroline.ewertsen@dadlnet.dk (C. Ewertsen), ferdinando. dambrosio@uniroma1.it (F. D'Ambrosio), peteris.prieditis@inbox.lv (P. Prieditis), salvatore.sorrenti@uniroma1.it (S. Sorrenti).
}

older than 40 years of age [1-4]. Most of them are hyperplastic nodules and not clinically relevant neoplasms [5]. However a proportion, although not at so high rates, are malignant within range $7-15 \%$ and/or become clinically significant and even fatal for the individual in less than $1 \%$ of cases [6] with a 2.4 times of increase in thyroid malignancy in the last 30 years [7]. Thyroid scintigraphy has a limited role in the evaluation of solitary thyroid nodules as the findings usually show relative uptake of the tracer within nodule with normal parenchyma overlay. Approximately $80 \%-85 \%$ of nodules are "cold" (lack of uptake) and 10\%-15\% of these are malignant [8]. A 99mTc pertechnetate scintigraphy can be used to differentiate "cold nodules" from autonomous functioning thyroid 
nodules (AFTN) or "hot nodules", in patients showing decreased serum thyrotropin (TSH) concentrations [9].

Ultrasound is the most common imaging modality to assess thyroid nodular lesions and to plan further treatment [5]. The first experience of image-guided radiofrequency ablation (RFA) with evaluation of efficacy and safety of ultrasound guided percutaneous RFA of thyroid nodules has been introduced and published in 2006 [10]. The results were positive - the volume of thyroid nodules significantly decreased and the majority of the patients reported an improvement of their symptoms. Since then the amount of publications about therapeutic options for thyroid nodules has grown noticeably.

The RFA method was considered an alternative to surgery in the beginning, but gradually the importance of RFA as a treatment option is growing for both - benign solid and partially cystic nodules and recurrent thyroid cancer [11,12], mainly because it is minimally invasive and some patients are ineligible for surgery $[11,13,14]$. In autonomously functioning thyroid nodules RFA can be considered as alternative to radioiodine therapy with the added benefit of not developing hypothyroidism [9]. RFA compared to other minimally invasive ablative techniques (e.g. microwave (MW), laser ablation (LA)), percutaneous ethanol injection (PEI) has been shown to be more effective in decreasing nodule mean volume [14], with small complication rates [15] and a marked improvement of nodule-related symptoms [11]. RFA procedure is highly operator dependent and practitioners experience is one of the crucial factors that affect treatment response [14].

The aim of this paper was to review the available literature on ultrasound guided RFA of thyroid nodules in order to evaluate indications, techniques, complications, limitations and outcome assessment.

\section{Methods}

The content, methodological strategy and the aims of the review were agreed before initiating the search.

\subsection{Search strategy}

A literature search was performed in Medline and Scopus in August 30, 2016.

The following search terms were applied, using Medline and MeSH terms: "thyroid gland", "thyroid neoplasms", "ultrasonography", "ablation techniques". To make sure that publications not yet indexed with MeSH terms were included, a free text search was included using the terms: "radiofrequency ablation", "RFA", "radiofrequency thermal ablation", "thyroid", "thyroid nodule", "ultrasound".

Two authors (MR and MR) reviewed all titles and abstracts.

The inclusion criteria were: original research papers in English on ultrasound guided RFA in thyroid nodules. Exclusion criteria were: non-human studies, case reports, review articles and metaanalyses, editorials, articles not written in English, studies with insufficient data; studies which compared RFA with other treatment methods.

All included papers were subsequently read by the three authors and consensus obtained through discussion. All reference lists were hand searched for further references. We registered: authors, year of publication, study design, sample size, nodule inclusion criteria (size, radioisotope scan, USG pattern, fluid component presence), procedure technical parameters, complications, follow-up period and volume reduction ratio (VRR). For malignant nodules - also pathological type and thyroglobulin $(\mathrm{Tg})$ level was evaluated.

\section{Results}

\subsection{Study selection and overview}

The procedure of study selection is presented in Fig. 1.

Four-hundred-and-fifteen publications were selected from titles and abstracts. 102 duplicates were removed from the titles and abstracts. Of the remaining 313 manuscripts, 24 were included.

16 manuscripts included data from benign nodules, 8 included data from malignant nodules (see Tables 1 and 2).

In total, out of 24 eligible studies RFA was used to treat 989 benign nodules ( 16 studies) in 895 patients, among them 79 nodules in 76 patients were treated with bipolar RFA ( 3 studies) and 300 malignant nodules ( 8 studies) in 241 patients.

12 studies were retrospective and 11 were prospective studies (two of these were randomized controlled trials (9.1\%) [16,17]).

RFA studies for benign nodules included only "cold" or nonfunctioning nodules in 9articles [10,16-23], 6 studies included also toxic (or "hot" in scintigraphy) and nonfunctioning nodules [9,24-29] and in one study authors did not mention functionality [30]. Ultrasound patterns of nodules varied - only solid or predominantly solid nodules were analyzed in 6 studies (solid portion $>50 \%$ ) $[16-18,20,25,26]$ and mixed studies with predominantly cystic or cystic nodules in addition to solid nodules in 8 studies $[9,10,19,22-24,29,30]$ and no information about patterns - in two studies $[21,28]$. The median diameter of the treated lesions was $3.3 \mathrm{~cm}$ (range $0.6-10.0 \mathrm{~cm}$ ) in 7studies that pointed out nodule diameter $[9,10,17,19,20,24,30]$. The detailed parameters are summarized in Tables 1 and 2

RFA studies for malignant nodules included two cases of medullary thyroid carcinoma [31,32], two studies included follicular carcinoma $[33,34]$ and one study reported on treatment of only papillary microcarcinomas [35]. Other treated malignancy histological variations were papillary thyroid carcinomas in 7 studies and exclusively in 3 studies [36-38]. The median nodule diameters before treatment were $10.95 \mathrm{~mm}(5.8-40 \mathrm{~mm})$, after treatment $2.2 \mathrm{~mm}$ (range $0.6-21.7 \mathrm{~mm}$ ).

Characteristics of procedure and results are discussed further in article.

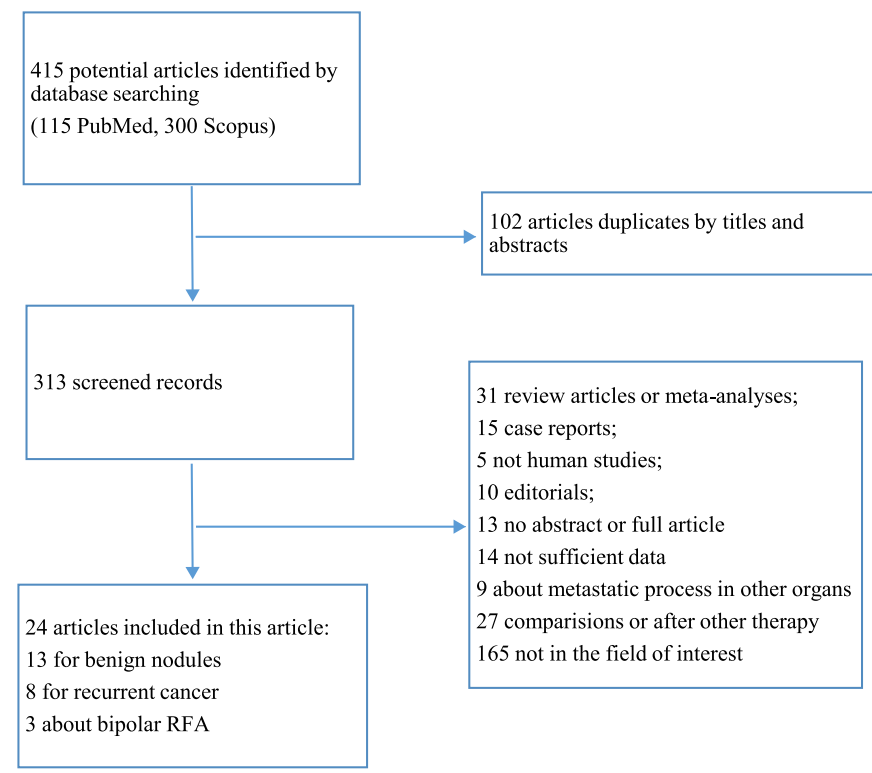

Fig. 1. Methodology of selection of analyzed publications. 
RFA for benign thyroid nodule.

\begin{tabular}{|c|c|c|c|c|c|c|c|c|c|c|c|c|c|}
\hline Author & Type & $\begin{array}{l}\text { No of } \\
\text { nodules } \\
\text { (patient) }\end{array}$ & Nodule size & $\begin{array}{l}\text { Radioisotope } \\
\text { scan }\end{array}$ & US pattern & $\begin{array}{l}\text { Fluid } \\
\text { component } \\
(\%)\end{array}$ & Electrode type & $\begin{array}{l}\text { No of } \\
\text { sessions }\end{array}$ & Time/power & $\begin{array}{l}\text { Complications } \\
\text { (resolve/do } \\
\text { not know or } \\
\text { not resolve }\end{array}$ & $\begin{array}{l}\text { Follow up } \\
\text { (months) }\end{array}$ & $\begin{array}{l}\text { Volume } \\
\text { reduction (\%) }\end{array}$ & Conclusion \\
\hline $\begin{array}{l}\text { Y. - S. Kim } \\
{[10]}\end{array}$ & Prospective & $35(30)$ & $\begin{array}{l}>1 \mathrm{~cm} \\
\text { mean } \\
2.66 \mathrm{~cm} \\
(1.1-5.6 \\
\mathrm{cm})\end{array}$ & Cold & $\begin{array}{l}9 \text { mainly solid; } \\
10 \text { mixed; } \\
16 \text { mainly cystic }\end{array}$ & Mean 58.4\% & $\begin{array}{l}\text { Internally } \\
\text { cooled } \\
\text { electrode, a } \\
17 \mathrm{~g} \text { straight } \\
\text { needle type } \\
\text { with a } 1 \mathrm{~cm} \\
\text { active tip }\end{array}$ & 1 & $\begin{array}{l}8-25 \min \\
(\text { mean } \\
15.1 \mathrm{~min}) / \mathrm{max} \\
44.4 \pm 10.1 \mathrm{~W} \\
(20-60 \mathrm{~W})\end{array}$ & $\begin{array}{l}1 \text { hematoma } \\
1 \text { I grade burn } \\
1 \text { mild- } \\
\text { moderate pain/ } \\
1 \text { ipsilateral } \\
\text { vocal cord palsy }\end{array}$ & $\begin{array}{l}6.4 \\
(1.1-18.5)\end{array}$ & $\begin{array}{l}72.98 \\
\text { (Residual V } \\
27.02 \% \pm 27.17 \% \text { ) }\end{array}$ & $\begin{array}{l}\text { RFA is effective, } \\
\text { ease of handling, } \\
\text { good control of } \\
\text { ablative lesion } \\
\text { etc. Excellent } \\
\text { alternative to } \\
\text { non-surgical and } \\
\text { surgical } \\
\text { therapeutic } \\
\text { modalities. }\end{array}$ \\
\hline $\begin{array}{l}\text { M. Deandrea } \\
{[24]}\end{array}$ & Prospective & $33(31)$ & $\begin{array}{l}>\mathbf{2} \mathbf{~ c m} \\
\text { median } \\
4.1 \mathrm{~cm} \\
(2.3-7.2)\end{array}$ & $\begin{array}{l}23 \text { hot } \\
10 \text { cold }\end{array}$ & $\begin{array}{l}18 \text { solid; } \\
15 \text { mixed }\end{array}$ & $<30 \%$ & $\begin{array}{l}14 \mathrm{~g} 9 \text {-prong } \\
\text { expandable } \\
\text { needle } \\
\text { electrode }\end{array}$ & 1 & $6-8 \mathrm{~min} /-$ & 3 edema/- & 6 & $\begin{array}{l}50.7 \pm 16.5 \\
\text { Hot nodules } \\
52.1 \% \pm 16.2 \% \text {; } \\
\text { Cold nodules } \\
46.2 \% \pm 16.9 \% \text {; }\end{array}$ & $\begin{array}{l}\text { RF was effective } \\
\text { and safe in } \\
\text { reducing volume } \\
\text { by about } 50 \% \text { and } \\
\text { compressive } \\
\text { symptoms in } \\
\text { large } \\
\text { benign nodules. } \\
\text { Hyperfunction } \\
\text { was fully } \\
\text { controlled in } 24 \% \\
\text { of patients and } \\
\text { partially reduced } \\
\text { in the others. }\end{array}$ \\
\hline $\begin{array}{l}\text { W. K. Jeong } \\
{[30]}\end{array}$ & Retrospective & $302(236)$ & $\begin{array}{l}>\mathbf{5} \mathbf{~ m m} \\
\text { mean } \\
2.44 \pm \\
1.36 \mathrm{~cm} \\
(0.6-10)\end{array}$ & - & $\begin{array}{l}164 \text { solid; } \\
89 \text { mixed; } \\
49 \text { cystic }\end{array}$ & & $\begin{array}{l}\text { Internally } \\
\text { cooled } \\
\text { electrode }(17 \mathrm{~g} \text {, } \\
\text { with } 1 \mathrm{~cm} \\
\text { active tip) }\end{array}$ & $1-6$ & $\begin{array}{l}5-30 \mathrm{~min} \\
(\text { mean } 14 \mathrm{~min}) / \\
20-70 \mathrm{~W}\end{array}$ & $\begin{array}{l}13 \text { pains } \\
>2 \text { days } \\
5 \\
\text { extrathyroidal } \\
\text { hematoma } \\
3 \text { voice } \\
\text { changes/- }\end{array}$ & $(1-41)$ & $\begin{array}{l}84.11 \pm 14.93 \% \\
(12.52-100)\end{array}$ & $\begin{array}{l}\text { RFA appears safe } \\
\text { and follow up } \\
\text { confirms volume } \\
\text { reduction. } \\
\text { (no nodule } \\
\text { volume increased } \\
\text { after RFA). }\end{array}$ \\
\hline $\begin{array}{l}\text { S. Spiezia } \\
{[25]}\end{array}$ & Prospective & $94(94)$ & $>4 \mathrm{ml}$ & $\begin{array}{l}28 \text { hot } \\
66 \text { cold }\end{array}$ & $\begin{array}{l}\text { Solid or } \\
\text { predominantly } \\
\text { solid. }\end{array}$ & $<30 \%$ & $\begin{array}{l}14 \mathrm{~g} 10 \mathrm{~cm} \text { long } \\
\text { needle with } 4 \\
\text { hooks } \\
\text { expandable to } \\
4 \mathrm{~cm} \text { (used max } \\
\text { to } 3.5 \mathrm{~cm} \text { ) }\end{array}$ & $1-3$ & $5-7 \mathrm{~min} /-$ & $\begin{array}{l}13 \text { pains }<24 \mathrm{~h} \\
5 \text { fever }-\end{array}$ & $(12-24)$ & $\begin{array}{l}79.4 \pm 2.5 \% \\
\text { (nontoxic }>\text { toxic) }\end{array}$ & $\begin{array}{l}\text { RFA offers } \\
\text { shrinkage of size, } \\
\text { relief of } \\
\text { symptoms and } \\
\text { autonomous } \\
\text { thyroid function. } \\
\text { It is attractive for } \\
\text { elderly patients. } \\
\text { Important to use } \\
\text { hook-umbrella } \\
\text { type needle for } \\
\text { large, solid TN. }\end{array}$ \\
\hline $\begin{array}{l}\text { J. H. Baek } \\
{[18]}\end{array}$ & Prospective & $\begin{array}{l}15(15) \\
+ \\
15 \text { control }\end{array}$ & $>2 \mathrm{~cm}$ & Cold & $\begin{array}{l}\text { Solidity - } \\
81.67 \pm \\
15.77 \% \\
(50-100 \%)\end{array}$ & $<50 \%$ & $\begin{array}{l}18 \mathrm{~g} 1 \mathrm{~cm} \text { active } \\
\text { tip internally } \\
\text { cooled } \\
\text { electrode }\end{array}$ & 1 & $\begin{array}{l}-1 \\
30-80 w\end{array}$ & & $\begin{array}{l}6.43 \pm \\
0.62(6-8)\end{array}$ & $79.7 \pm 14.6 \%$ & $\begin{array}{l}\text { RFA is effective } \\
\text { for reducing } \\
\text { nodule volume } \\
\text { and relieving } \\
\text { nodule-related } \\
\text { clinical problems } \\
\text { and that an effect } \\
\text { due to } \\
\text { spontaneous }\end{array}$ \\
\hline
\end{tabular}




\begin{tabular}{|c|c|c|c|c|c|c|c|c|c|c|c|c|c|}
\hline & & & & & & & & & & & & & $\begin{array}{l}\text { a similar control } \\
\text { group. }\end{array}$ \\
\hline $\begin{array}{l}\text { A. Faggiano } \\
{[26]}\end{array}$ & Prospective & $\begin{array}{l}20(20) \\
+ \\
20 \text { control }\end{array}$ & $>4 \mathrm{ml}$ & $\begin{array}{l}- \\
10 \text { toxic; } \\
10 \text { nontoxic }\end{array}$ & $\begin{array}{l}\text { Solid or } \\
\text { predominantly } \\
\text { solid }\end{array}$ & $<30 \%$ & $\begin{array}{l}14 \mathrm{~g} 10 \mathrm{~cm} \text { long } \\
\text { and made of } 4 \\
\text { hooks } \\
\text { expandable to } \\
4 \mathrm{~cm} \text { (used max } \\
\text { to } 3.5 \mathrm{~cm} \text { ). }\end{array}$ & & $5-7 \mathrm{~min} /$ & - & $9-12$ & $84.9 \pm 1.5$ & $\begin{array}{l}\text { RTA induced a } \\
\text { marked TN } \\
\text { volume shrinkage } \\
\text { resulting in } \\
\text { parallel } \\
\text { improvement of } \\
\text { pressure } \\
\text { symptoms. } \\
\text { Inmost patients } \\
\text { with toxic TNs, } \\
\text { hyperthyroidism } \\
\text { significantly } \\
\text { improved as well. }\end{array}$ \\
\hline $\begin{array}{r}\text { H. K. Lim } \\
(2012)\end{array}$ & Retrospective & $126(111)$ & $\begin{array}{l}>\mathbf{2} \mathbf{c m} \\
3.3 \pm 1 \mathrm{~cm} \\
(2-6 \mathrm{~cm})\end{array}$ & $\begin{array}{l}- \\
\text { (non - } \\
\text { functioning) }\end{array}$ & $\begin{array}{l}81 \text { solid } \\
(>50 \%) ; \\
45 \text { mixed } \\
(\text { solid }=<50 \%)\end{array}$ & $\begin{array}{l}65.1= \\
/-32.9 \\
(5-100)\end{array}$ & $\begin{array}{l}17-\text { or } 18-\mathrm{g} \\
\text { internally } \\
\text { cooled } \\
\text { electrode with } \\
1,1.5-, 2 \text {-cm } \\
\text { active tips }\end{array}$ & $\begin{array}{l}2.2 \pm \\
1.4(1-7)\end{array}$ & $\begin{array}{l}-l \\
30-120 \mathrm{~W}\end{array}$ & $\begin{array}{l}1 \text { hematoma } \\
1 \text { vomiting } \\
2 \text { local pain } \\
1 \text { voice changes } \\
1 \text { brachial } \\
\text { plexus injury/- }\end{array}$ & $\begin{array}{l}49.4 \pm \\
13.6(36-81)\end{array}$ & $\begin{array}{l}93.5 \pm 11.7 \% \text { (17 } \\
-100 \% \text { ) } \\
\text { (complete } \\
\text { disappearance } \\
18.3 \% \text { ) } \\
\text { (recurrence rate } \\
5.6 \% \text { ) }\end{array}$ & $\begin{array}{l}\text { RFA is effective in } \\
\text { shrinking benign } \\
\text { TN as well as in } \\
\text { controlling } \\
\text { nodule - related } \\
\text { problems } \\
\text { without life - } \\
\text { threatening } \\
\text { complications or } \\
\text { sequelae. }\end{array}$ \\
\hline $\begin{array}{l}\text { E. J. Ha } \\
{[20]}\end{array}$ & Retrospective & $\begin{array}{l}14(11) \\
\text { with } \\
\text { previous } \\
\text { lobectomy }\end{array}$ & $\begin{array}{l}2.9 \pm 2.4 \mathrm{~cm} \\
(1.5-6 \mathrm{~cm})\end{array}$ & $\begin{array}{l}- \\
\text { (nontoxic - } \\
\text { TSH \& T3, } \\
\text { T4 normal) }\end{array}$ & Solid (>50\%) & $<50 \%$ & $\begin{array}{l}18 \mathrm{~g} 1 \mathrm{~cm} \text { active } \\
\text { tip internally } \\
\text { cooled } \\
\text { electrode }\end{array}$ & 1 & $\begin{array}{l}-1 \\
-\end{array}$ & - & $\begin{array}{l}43.7 \pm \\
30.7(7-92)\end{array}$ & $87.2 \%$ & $\begin{array}{l}\text { In patients with } \\
\text { previous } \\
\text { lobectomy, RFA } \\
\text { have advantages } \\
\text { over surgery or } \\
\text { RAI therapy in } \\
\text { terms of } \\
\text { maintaining } \\
\text { thyroid function. }\end{array}$ \\
\hline $\begin{array}{l}\text { G. Turtulici } \\
{[21]}\end{array}$ & Retrospective & $45(45)$ & $>12 \mathrm{ml}$ & Nonfunctioning & - & - & $\begin{array}{l}18 \mathrm{~g}, 70-\mathrm{mm} \text {, } \\
\text { fixed RF } \\
\text { electrode with } \\
10 \mathrm{~mm} \text { active } \\
\text { tip, } \\
\text { synchronized } \\
\text { with tracking } \\
\text { device } \\
\text { - > locating the } \\
\text { exact position } \\
\text { of electrode } \\
\text { and projecting } \\
\text { its path on } \\
\text { Bmode image. }\end{array}$ & 1 & $\begin{array}{l}- \\
70-80 \mathrm{~W} \text { in } \\
\text { center, } 30 \\
-40 \mathrm{~W} \text { in } \\
\text { proximity }\end{array}$ & $\begin{array}{l}1 \text { first-grade } \\
\text { skin burn/- }\end{array}$ & 6 & $\begin{array}{l}72.6 \pm 11.3 \% \\
\text { (without } \\
\text { recurrence) }\end{array}$ & $\begin{array}{l}\text { The VT system } \\
\text { provides effective } \\
\text { guidance in RF } \\
\text { ablation of } \\
\text { benign thyroid } \\
\text { nodules. This } \\
\text { technique } \\
\text { may improve the } \\
\text { accuracy and } \\
\text { outcome of RF } \\
\text { ablation while } \\
\text { reducing the } \\
\text { complication rate } \\
\text { and shortening } \\
\text { the } \\
\text { learning curve for } \\
\text { this procedure. } \\
\text { inued on next page) }\end{array}$ \\
\hline
\end{tabular}




\begin{tabular}{|c|c|c|c|c|c|c|c|c|c|c|c|c|c|}
\hline Author & Type & $\begin{array}{l}\text { No of } \\
\text { nodules } \\
\text { (patient) }\end{array}$ & Nodule size & $\begin{array}{l}\text { Radioisotope } \\
\text { scan }\end{array}$ & US pattern & $\begin{array}{l}\text { Fluid } \\
\text { component } \\
\text { (\%) }\end{array}$ & Electrode type & $\begin{array}{l}\text { No of } \\
\text { sessions }\end{array}$ & Time/power & $\begin{array}{l}\text { Complications } \\
\text { (resolve/do } \\
\text { not know or } \\
\text { not resolve }\end{array}$ & $\begin{array}{l}\text { Follow up } \\
\text { (months) }\end{array}$ & $\begin{array}{l}\text { Volume } \\
\text { reduction (\%) }\end{array}$ & Conclusion \\
\hline $\begin{array}{l}\text { R. Cesareo } \\
{[16]}\end{array}$ & $\begin{array}{l}\text { Prospective } \\
\text { (randomized, } \\
\text { controlled) }\end{array}$ & $\begin{array}{l}2(42) \\
+ \\
42(42) \\
\text { control }\end{array}$ & $\begin{array}{l}>\mathbf{2} \mathbf{~ c m} />5 \\
\mathrm{ml}\end{array}$ & Cold & $\begin{array}{l}\text { Solid or } \\
\text { predominantly } \\
\text { solid (>70\%) }\end{array}$ & $<30 \%$ & $\begin{array}{l}17 \mathrm{~g}, 15 \mathrm{~cm} \\
\text { electrode with } \\
1 \mathrm{~cm} \text { active tip }\end{array}$ & 1 & $\begin{array}{l}37 \pm 17.4 \mathrm{~min} \\
(17-85) / 60 \mathrm{~W}\end{array}$ & $\begin{array}{l}2 \text { voice } \\
\text { changes/ } \\
1 \text { permanent } \\
\text { right } \\
\text { paramedian } \\
\text { vocal cord palsy } \\
\text { with } \\
\text { inspiratory } \\
\text { stridor without } \\
\text { dysphonia }\end{array}$ & 6 & $68,6 \pm 13,5 \%$ & $\begin{array}{l}\text { RFA is effective in } \\
\text { reducing thyroid } \\
\text { nodule volume. } \\
\text { The best } \\
\text { reduction rate } \\
\text { was observed in } \\
\text { small TNs. The } \\
\text { thyroid } \\
\text { volumetric } \\
\text { reduction does } \\
\text { not change } \\
\text { according to the } \\
\text { sonographic } \\
\text { features. The } \\
\text { mean treatment } \\
\text { duration was } \\
\text { longer in larger } \\
\text { TNs. }\end{array}$ \\
\hline $\begin{array}{l}\text { M. Deandrea } \\
{[17]}\end{array}$ & $\begin{array}{l}\text { Prospective } \\
\text { (randomized, } \\
\text { controlled) } \\
\text { (international } \\
\text { - Korea vs. } \\
\text { Italy) }\end{array}$ & $\begin{array}{l}40(40) \\
+ \\
40(40) \\
\text { control }\end{array}$ & $\begin{array}{l}\mathbf{1 0}-\mathbf{2 0} \mathrm{ml} \\
\text { mean } 4 \mathrm{~cm}\end{array}$ & Nonfunctioning & Solid (>70\%) & $<30 \%$ & $\begin{array}{l}\text { Single hook } \\
18 \text { g internally } \\
\text { cooled } \\
\text { electrode }\end{array}$ & 1 & $\begin{array}{l}\text { It: } \\
819.5 \pm 225.9 \mathrm{~s} / \\
\text { mean } \\
49.7 \pm 4.7 \mathrm{~W} \\
\text { Ko: } \\
435.8 \pm 142.4 \mathrm{~s} / \\
\text { mean } \\
75.3 \pm 10.4 \mathrm{~W}\end{array}$ & - & 6 & $71 \%$ & $\begin{array}{l}\text { RFA was effective } \\
\text { in reducing the } \\
\text { volume. The } \\
\text { outcome was } \\
\text { similar in centers } \\
\text { with different } \\
\text { experience in the } \\
\text { moving-shot } \\
\text { technique. }\end{array}$ \\
\hline $\begin{array}{l}\text { J. Y. Sung } \\
\text { [9] - RCT }\end{array}$ & $\begin{array}{l}\text { Retrospective, } \\
\text { multicenter }\end{array}$ & $44(44)$ & $\begin{array}{l}\text { Mean } 3.8 \\
\pm 1.4 \mathrm{~cm} \\
(1.3- \\
9 \mathrm{~cm}) \\
\text { Mean V } \\
18.5 \pm 30.1 \\
\mathrm{ml}(0.5- \\
174.3 \mathrm{ml})\end{array}$ & $\begin{array}{l}23 \text { toxic, } \\
21 \text { pretoxic }\end{array}$ & $\begin{array}{l}26 \text { solid, } \\
16 \text { predominantly } \\
\text { solid, } \\
2 \text { predominantly } \\
\text { cystic }\end{array}$ & - & $\begin{array}{l}\text { Internally } \\
\text { cooled } \\
\text { electrode of } \\
7 \mathrm{~cm} \text { length, } \\
18 \mathrm{G}, 0.5-, 1-\text {, } \\
1.5-\mathrm{cm} \text { active } \\
\text { tip }\end{array}$ & $\begin{array}{l}1.8 \pm 0.9 \\
(1-6)\end{array}$ & $\begin{array}{l}12 \pm 5.9 \mathrm{~min} \\
(2.5-30 \mathrm{~min}) / \\
63.3 \pm 26.3 \mathrm{~W} \\
(20-120 \mathrm{~W})\end{array}$ & - & $\begin{array}{l}19.9 \pm \\
12.6 \mathrm{mo} \\
(6-56 \mathrm{mo})\end{array}$ & $81.7 \pm 13.6 \%$ & $\begin{array}{l}\text { RFA can be } \\
\text { considered a } \\
\text { possible } \\
\text { alternative to } \\
\text { surgery or } \\
\text { radioiodine } \\
\text { therapy without } \\
\text { development of } \\
\text { hypothyroidism. }\end{array}$ \\
\hline $\begin{array}{l}\text { E. Aysan } \\
\text { [22] }\end{array}$ & Prospective & $100(100)$ & $\begin{array}{l}>\mathbf{1} \mathbf{~ c m} \\
\text { mean } 16.8 \\
\text { ml }(0.52- \\
220.89 \mathrm{ml})\end{array}$ & $\begin{array}{l}- \\
\text { (euthyroid) }\end{array}$ & $\begin{array}{l}51 \text { solid (>80\%); } \\
35 \text { mixed; } \\
14 \text { cystic }(>80 \%)\end{array}$ & - & $\begin{array}{l}\text { Internally } \\
\text { cooled } 18 \mathrm{~g} \text {, } \\
70 \mathrm{~mm} \text { length, } \\
10 \mathrm{~mm} \text { active } \\
\text { tip electrode }\end{array}$ & 1 & $\begin{array}{l}9.5 \mathrm{~min}(4 \\
-22.5 \mathrm{~min}) / \\
\text { standardized } \\
70 \mathrm{~W}\end{array}$ & $\begin{array}{l}1 \text { temporary } \\
\text { hoarseness/- }\end{array}$ & $15.4(6-24)$ & 84.5 & $\begin{array}{l}\text { RFA is with } \\
\text { minimal } \\
\text { invasiveness and } \\
\text { a low } \\
\text { complication } \\
\text { rate. In a cystic } \\
\text { type nodules, the } \\
\text { success rates are } \\
\text { greater. General } \\
\text { anesthesia can be } \\
\text { a good option for } \\
\text { anxious cases to } \\
\text { gather better } \\
\text { results. }\end{array}$ \\
\hline
\end{tabular}



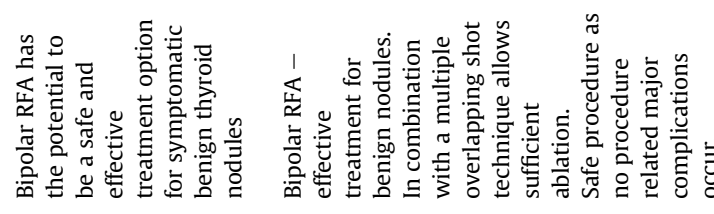$$
\text { . }
$$$$
\text { ڤ̊ }
$$

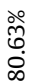$$
\text { 苞 }
$$
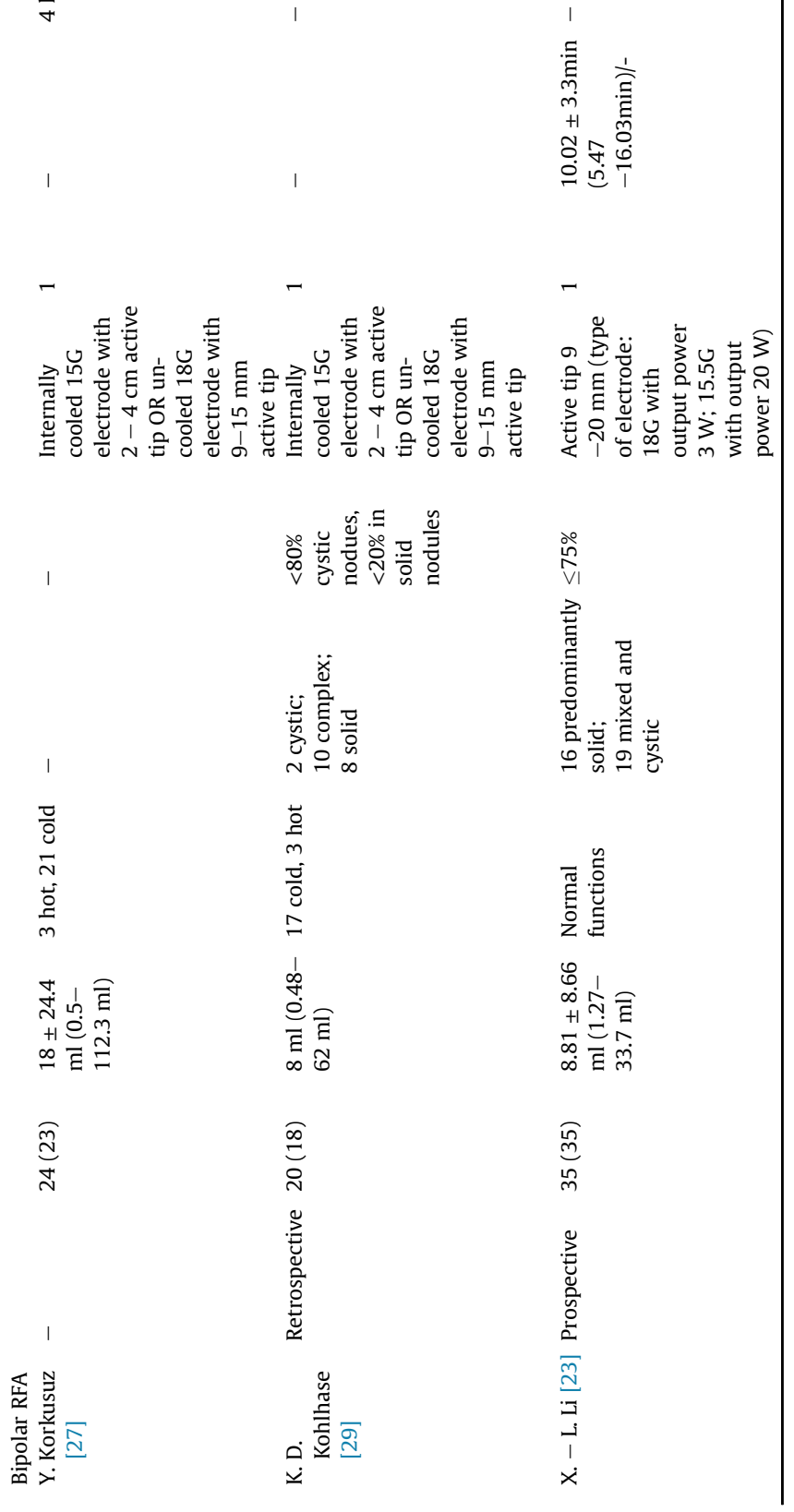

\section{Nodule criteria}

\subsection{Benign nodules}

Main indications for RFA procedure in patients with benign thyroid nodules include nodule - related clinical problems [12]. However, many of the analyzed studies chose different morphological and functional criteria as follows: (1) cosmetic and/or symptomatic nodules; (2) cytologically confirmed benign nodule on two subsequent US - guided FNAB; (3) US findings without suspicious malignant features; (4) refusal of or ineligible for surgery; (5) treatment for the thyroid nodule six months prior to RFA therapy; (6) adult patients ( $>18$ years) [see benign nodule table].

\subsubsection{Exclusion criteria}

Main exclusion criteria were US features suggestive of malignancy and various previous therapies for thyroid nodules $[17,30]$. RFA does not affect thyroid function in patients with previous lobectomy [20].

\subsubsection{Nodule size}

One of the most important criteria for nodule selection is thyroid nodule size. The largest diameter in most of studies was at least $2 \mathrm{~cm}[15,16,18,19,24]$. However, one study included nodules with size from $1 \mathrm{~cm}$ [22], that may be explained by evolving technique with more experience.

\subsubsection{Nodule volume}

Other studies used nodule volume as criteria for inclusion parameter with variability from 4 to $12 \mathrm{ml}$ in published studies $[16,17,21,26]$. Based on the fact that nodule gradual volume reduction is used as the criteria of effectiveness after RFA [39], it would be more accurate to use volume as an inclusion criterion as well.

There have been discussions about how initial nodule size or volume affects therapy results and it has been considered that RFA gives more effective results if nodule volume is less than $20 \mathrm{ml}$ $[11,14]$. Patients with larger nodules may require more than one session or, if it is possible, preferably surgery as the first - line treatment option [14].

\subsubsection{US findings}

Ultrasound pattern of nodule - solid, mixed or cystic - is an important factor for short-term results. Rapid shrinkage was more typical for predominantly cystic nodule, although long term data suggested no significant difference of thyroid volumetric reduction between solid, predominantly solid nodules and cystic nodules $[14,18]$.

\subsubsection{Laboratory findings}

Serum thyroid hormone and thyrotropin levels were measured in the majority of studies and according to study type, functional, non-functional or both types of nodules were selected. There are fewer studies with functional nodules (or also called "hot" at scintigraphy) due to risk of the abrupt changes in functional state and incomplete response to treatment and recurrence, that may be explained by the insufficient ablation of the nodule margins [14].

\subsection{Malignant nodules and recurrent carcinoma}

RFA can be used to treat both benign thyroid nodules and recurrent thyroid cancers but it is not established as a first line treatment for thyroid carcinomas [14,24]. Some authors suggest that for nodules with Bethesda categories 3, 4, 5 and 6, the RFA should be avoided because it does not resolve patient's problems, delays surgery and neglects undetectable nodal metastases [40,41]. 
Table 2

\begin{tabular}{|c|c|c|c|c|c|c|c|c|c|c|c|c|}
\hline Author (y) & Study type & $\begin{array}{l}\text { Patient } \\
\text { (nodule } \\
\text { count) }\end{array}$ & $\begin{array}{l}\text { Pathological } \\
\text { type }\end{array}$ & Sessions & RFA time & $\begin{array}{l}\text { Volume } \\
\text { (mm3) } \\
\text { bef/af }\end{array}$ & $\begin{array}{l}\text { Diameter } \\
(\mathrm{mm}) \\
\text { bef/af }\end{array}$ & $\begin{array}{l}\text { Tg le }(\mathrm{ng} / \mathrm{ml}) \\
\text { bef/af }\end{array}$ & $\begin{array}{l}\text { Follow - } \\
\text { up (mo) }\end{array}$ & VRR & Prognosis & Complications \\
\hline $\begin{array}{l}\text { Monchik, J. M. } \\
\text { [31] }\end{array}$ & Retrospective & $16(22)$ & $\begin{array}{l}15 \text { papillary TC, } \\
1 \text { medullary TC }\end{array}$ & $1-6$ & $2-12 \mathrm{mn}$ & NA & $\begin{array}{l}17(8-40) / \\
\mathrm{NA}\end{array}$ & $37 / 1.9$ & $\begin{array}{l}40.7 \\
(10-68)\end{array}$ & $\begin{array}{l}\text { Decrease } \\
\text { in size } \\
\sim 95 \%\end{array}$ & 2 recurrent & $\begin{array}{l}1 \text { hoarseness, } \\
1 \text { skin burn }\end{array}$ \\
\hline $\begin{array}{r}\text { Baek, J. H. } \\
{[36,44]}\end{array}$ & Retrospective & $10(12)$ & Papillary TC & $1.2(1-2)$ & $\begin{array}{l}402.5 \mathrm{~s} \\
(60-900)\end{array}$ & $\begin{array}{l}55.5 \pm \\
50.3 / 5.7 \\
\pm 9.3\end{array}$ & $\begin{array}{l}13.8 \pm 7 / 3.3 \\
\pm 3.9\end{array}$ & $\begin{array}{l}8.1 \pm 11.8 / \\
2.2 \pm 3.3\end{array}$ & $\begin{array}{l}23 \pm 5.5 \\
(16-31)\end{array}$ & $\begin{array}{l}93 \%(49- \\
100 \%)\end{array}$ & $\begin{array}{l}2 \mathrm{p} \text { new metastatic } \\
\text { tumors } \\
\text { (92\% therapeutic } \\
\text { success rate; } 50 \% \\
\text { complete } \\
\text { disappearance) }\end{array}$ & 1 dysphonia \\
\hline $\begin{array}{l}\text { Park, K. W. } \\
\text { [33] }\end{array}$ & Prospective & $11(16)$ & $\begin{array}{l}15 \text { papillary TC, } \\
1 \text { follicular TC }\end{array}$ & 1 & $\begin{array}{l}11.7 \min \\
(1-22)\end{array}$ & $\begin{array}{l}8493.75 \\
\pm 9714.15 / \\
5180.67 \pm \\
7034.41\end{array}$ & $\begin{array}{l}27.6 \pm \\
13.1 / 21.7 \\
\pm 13.5\end{array}$ & $\begin{array}{l}\text { Decrease } 58.4 \% \\
(40.8-81.4 \%) \text { in } \\
9 p, 3 p \\
\text { increased } \mathrm{Tg}\end{array}$ & $6(1-14)$ & $\begin{array}{l}50.9 \% \\
(9.4-96.8 \%)\end{array}$ & $\begin{array}{l}2 \text { regrowth } \\
1 \text { died (respiratory } \\
\text { failure) }\end{array}$ & $\begin{array}{l}1 \text { 2nd degree skin } \\
\text { burn } \\
11 \text { self-limited neck } \\
\text { swelling and } \\
\text { discomfort }\end{array}$ \\
\hline $\begin{array}{l}\text { Guenette, J. P. } \\
\text { [34]/RFA + PEI }\end{array}$ & Retrospective & $14(21)$ & $\begin{array}{l}\text { Papillary TC, } \\
\text { Follicular TC }\end{array}$ & $\mathrm{NA}$ & $2-12 \mathrm{~min}$ & NA & $\begin{array}{l}15(5-37) \\
\text { NA }\end{array}$ & $\begin{array}{l}38.6 \pm 49.89 / \\
1.49 \pm 1.41\end{array}$ & $\begin{array}{l}61.3 \\
(10-127)\end{array}$ & NA & No progression & $\begin{array}{l}1 \text { permanent vocal } \\
\text { cord paralysis }\end{array}$ \\
\hline Lim, H. K. (2014) & Retrospective & $39(61)$ & Papillary TC & $\begin{array}{l}1.1 \pm 1.3 \\
(1-2)\end{array}$ & $\begin{array}{l}243.5 \pm \\
264.7 \mathrm{sec} \\
(33-1200 \mathrm{sec})\end{array}$ & $\begin{array}{l}200 \pm \\
350 / 20 \pm \\
110\end{array}$ & $\begin{array}{l}7.9 \pm 4.3 / \\
1.1 \pm 3.0\end{array}$ & $\begin{array}{l}1.21 \pm 1.91 / \\
0.5 \pm 0.8(\mathrm{mIU} / \\
\mathrm{L})\end{array}$ & $\begin{array}{l}26.4 \pm 13.7 \\
(6-48)\end{array}$ & 95.1 & $\begin{array}{l}82 \% \text { completely } \\
\text { disappear }\end{array}$ & $\begin{array}{l}3 \text { voice change } \\
\text { (recovered) } \\
4 \text { local pain during } \\
\text { RFA } \\
\text { Overall } 7.7 \%\end{array}$ \\
\hline Lee, S. J. [32] & Retrospective & $32(34)$ & $\begin{array}{l}34 \text { papillary TC, } \\
1 \text { medullary TC }\end{array}$ & $\begin{array}{l}1.3 \text { (range } \\
1-3)\end{array}$ & $\begin{array}{l}4.4 \text { (40sec- } \\
15 \mathrm{~min})\end{array}$ & $\begin{array}{l}173.9 \pm \\
198.7 / 6.2 \\
\pm 27.9\end{array}$ & $\begin{array}{l}8.1 \pm 3.4 / \\
0.6 \pm 1.8\end{array}$ & $\begin{array}{l}16.7 \pm 32.3 / \\
10.5 \pm 26.3\end{array}$ & $30(6-49)$ & 96.4 & $\begin{array}{l}\text { Not recurrent }(1 \mathrm{p} \\
\text { excluded }- \\
\text { surgery }=93.3 \% \\
\text { complete } \\
\text { disappear) }\end{array}$ & $\begin{array}{l}6 \text { voice change (5 } \\
\text { recovered) }\end{array}$ \\
\hline Kim, J. - H. [38] & Retrospective & $27(36)$ & Papillary TC & $1.1(1-2)$ & $\begin{array}{l}5.5 \pm 1.5 \min \\
(3-8 \mathrm{~min})\end{array}$ & $\begin{array}{l}191.8 \pm \\
273.3 / 8.6 \\
\pm 45.5\end{array}$ & $\begin{array}{l}(<2 \mathrm{~cm}) \\
9.8 \pm 3.6 / \\
\text { NA }\end{array}$ & $\begin{array}{l}1.68 \pm 3.13 / \\
0.9 \pm 1.44\end{array}$ & $\begin{array}{l}32.4 \pm \\
11.1\end{array}$ & $>70 \%$ & $\begin{array}{l}3 p(11.5 \%) \\
\text { recurrent }\end{array}$ & $\begin{array}{l}1 \mathrm{p} \text { transient } \\
\text { hoarseness }(3.7 \%)\end{array}$ \\
\hline Zhang, M. [35] & Prospective & $92(98)$ & $\begin{array}{l}\text { Papillary } \\
\text { microcarcinoma }\end{array}$ & 1 & $\begin{array}{l}450.8 \pm \\
230.2 \mathrm{~s} \\
(91-902 \mathrm{sec})\end{array}$ & $\begin{array}{l}118.8 \pm \\
106.9 / 9.9 \\
\pm 19\end{array}$ & $\begin{array}{l}5.8 \pm 2.2 / \\
\mathrm{NA}\end{array}$ & - & $\begin{array}{l}7.8 \pm 2.9 \\
(3-18)\end{array}$ & $96 \%$ & No recurrent & No major \\
\hline
\end{tabular}


The recent study by Zhang et al., 2016 used US guided radiofrequency ablation to treat low-risk papillary thyroid microcarcinomas (PTMC) with good prognosis that are considered as surgically overtreated. Using US and contrast-enhanced ultrasound (CEUS) examinations before ablation, all 92 cases were confirmed by core biopsy before and after treatment, and authors revealed that RFA can effectively eliminate low-risk PTMC with no signs of recurrence or residual tumor up to 12-18 months follow up period.

There were few studies about RFA effectiveness in recurrent thyroid carcinomas and the limitations here are the retrospective study type (only two prospective research studies have been found to the best of our knowledge [33,35]) and the limited patient population evaluated. In cases of recurrent thyroid carcinoma surgical treatment can be affected by scar tissue formation from the previous surgery and also the patient's clinical condition. Scar tissue may cause marked distortion of the normal anatomy of the neck, making reoperation difficult even for experienced surgeons and it is associated with a high rate of morbidity [31]. Therefore, besides surgery and active surveillance, RFA might represent an alternative for managing PTMCs, especially in patients ineligible for surgery.

\section{Technical parameters}

At present, there are two available RFA systems - monopolar and bipolar. Monopolar RFA system has been used for 10 years and the majority of publications are about its efficiency, whereas bipolar system has been recently introduced to avoid some of disadvantages of the monopolar RFA approach.

RFA procedure requires a radiofrequency generator and electrodes. Monopolar electrodes used in RFA can be divided in two types - single active tip and expandable several hook/prong/tine electrodes. Each of these electrodes has distinctive advantages.

Studies that used multi-tined expandable electrode declared following benefits: (1) treatment of larger areas in a single session [24,25]; (2) less traumatic for larger area compared with smaller electrode, which require multiple sessions [24]. However, authors admit some disadvantages for multiple tines -difficult to monitor, cannot be used in small nodules, needle is slightly larger (14G) and therefore more traumatic for tissue and may need incision before insertion [24-26]. In addition, the treatment may not be distributed to the entire lesion [25]. Based on this, several hook electrodes are advisable only for large nodules (at least $4 \mathrm{ml}$ [25]) and in cases where only a single treatment session is required [26].

Single active tip internally cooled electrode is used more often. Needle is smaller (17-18G) with variable active tip length - mostly $1 \mathrm{~cm}$, but in some studies also $1.5 \mathrm{~cm}$ or $2 \mathrm{~cm}$ [19]. Straight, internally cooled electrode can be used for smaller nodules because it is easier to monitor the electrode position and to evaluate ablation extent. This approach allows to ablate the periphery of the nodule more precisely and to avoid marginal regrowth. This electrode is also advisable for nodules with cystic components [25]. When dealing with hard nodules, electrode can bend considerably, producing inaccuracies in positioning of the tip [21].

\section{Procedure technique}

At the beginning of the procedure, patient is in the supine position with mild neck extension and operator evaluates nodule position and selects the most adequate approach. It is difficult to carry out the procedure if the nodule is in the critical position or the patient has an enlarged thyroid gland with retrosternal growth [28]. Local anesthetic is the most often used method of anesthesia used during the procedure, although there are some studies in which intravenous pain relief medication or even general anesthesia are used [22].

\subsection{Trans-isthmic approach}

Since Jeong et al. (2008) has proposed to use the trans-isthmic approach, most of studies have selected this application. In transisthmic approach electrode passes through sufficient thyroid parenchyma thereby preventing a change in the position of electrode active tip and avoiding damages of "danger triangle" (including the recurrent laryngeal nerve and/or the esophagus). Also, when treating mixed or mainly cystic nodules, secure positioning of the electrode prevents leakage of ablated hot fluid outside the thyroid gland tissue.

Rarely, if trans-isthmic approach is not possible, cranio-caudal approach can be used in which the operator inserts the electrode tip along the long axis of the nodule. This method has several disadvantages: (1) visualization of the entire electrode is difficult; (2) the electrode may accidently reposition during procedure due to patient talking or swallowing movements [18].

\subsection{Time and power}

RFA procedure duration depends on nodule size and structure, with the shortest described period being $2.5 \mathrm{~min}$ [9]. Pooled time IQR for benign nodules is $9.2 \mathrm{~min}(11.4-20.6 \mathrm{~min})$ [see benign nodule table].

Ablation starts with 20-30 W of RF power and if the transient hyperechoic zone at the electrode tip does not appear, the power could be gradually increased in 5 to 10Wincrements, reaching full capacity even at $120 \mathrm{~W}[9,19]$. In a different strategy RF power range from 70 till $80 \mathrm{~W}$ is used in the center of the nodule and from 30 till $40 \mathrm{~W}$ in the proximity of the nodule margins [21]. The RFA is terminated when all conceptual units have become as transient hyperechoic zones or if the patient cannot tolerate the pain. In most studies in the latter case, the ablation is just temporarily stopped and resumed after the pain has been relieved [see benign nodule table].

\subsection{Fixed electrode or moving shot technique}

Fixed electrode technique with monopolar electrode for thyroid nodules is not applicable because it is considered dangerous to the surrounding important structures, e.g. esophagus and recurrent laryngeal nerve. Instead -a moving shot technique is used -initially the electrode tip is positioned in the deepest conceptual ablation unit of a thyroid nodule and RFA is performed unit-by-unit, continuously moving tip backwards and in the superficial direction [18].

\subsection{Multiple overlapping shots technique}

Bipolar RFA is a new method and there are just few studies published recently about it [23,28,29]. In monopolar systems induced current flows between the grounding pads and the probe, therefore some amount of energy is lost to the penetrated tissues. In bipolar systems both the electrodes are in the probe and less electric current is lost. Therefore, Kohlhase et al. (2016) suggested new technique - multiple overlapping shots technique ("MOST") based on fixed shot technique. In case of bipolar RFA, the ablation zone expands spherically and allows larger ablation even if probe is not moved. As authors note, this technique requires less technical skill and therefore is recommended for nodules adjacent to vulnerable structures.

Overall, bipolar RFA procedure may provide a more predictable ablation zone in terms of size and geometric shape. A higher energy 
density per nodule and a shorter duration of ablation time are beneficial both for the patient satisfaction and for the long term results [28]. However, further investigation is needed to calculate the requisite energy transfer to achieve ablation [28].

\subsection{Number of sessions}

The number of sessions is more crucial for large nodules with solid structure than for mixed or cystic nodules. Number of sessions significantly increase as the initial nodule volume increase [19]. On average, one session is used and there are no sufficient data that shows worse volume reduction ratio for one session approach $[10,16-18,20-22,24,26]$.

\subsection{Energy per nodule}

Delivered energy per nodule volume unit is the most important reported factor for a long-term shrinkage [17]. Some studies have proved that the more intense and shorter treatment sessions are, the quicker shrinkage is obtained in comparison to less powerful and longer treatment [26]. On the other hand, one of the largest and most recent international prospective randomized controlled trials [17] suggested evaluating tumor shrinkage by the delivered energy per $\mathrm{ml}$ of the treated tissue, while the time of treatment and the power were not crucial.

\subsection{Precautions}

If the nodule has a cystic component, it is advised to aspirate at least half of the fluid before RFA to avoid the possibility of cystic fluid leakage resulting from the increased pressure during procedure [10].

Complete ablation of the periphery of the nodule is important in order to prevent marginal regrowth [19]. However, regions next to critical structures may remain undertreated. One of the possible options for challenging cases to improve monitoring of the electrode active tip during placement and ablation are virtual needle tracking systems. These tracking systems enable electrode positioning out-of-US imaging plane approach that may be required in certain situations (e.g. deep nodule localization, poorly detectable danger triangle) [21].

During the procedure and at least $1-2 \mathrm{~h}$ after RFA the patient needs to be strictly observed in order to be treated in case of severe neck pain or discomfort, with oral or intravenous analgesic drugs.

\subsection{Procedure for malignant nodule}

RFA procedure technique slightly differs for recurrent thyroid cancer nodules. The same RFA generators are used but modified electrodes are available that are shorter (7-cm shaft length), thinner (18G) and with smaller active tip (0.5-, 0.7-cm). This type of electrode can easily penetrate small metastatic tumors in the previous surgical scarring areas, it decreases the risk of burning and injury to normal tissue and it is easier to control [36]. It has been recommended to use the same trans-isthmic approach and moving shot technique although most of studies add hydrodissection technique - injection of lidocaine and dextrose solution between the tumor and the expected location of nerves and other critical structures to prevent thermal injury of them [32,36-38]. There is no constancy with procedure duration of RFA in malignant nodule group.

For most of nodules one RFA session was performed but range is wide - up to 6 sessions per nodule. The number of session depends on nodule size, position, tumor multiplicity and patient's tolerance of pain.

\section{Complications}

Awareness of the possible complications with different techniques is very important [39]. Complications are subdivided in minor and major events. In a recent multicenter study of 1459 patients with benign thyroid nodules, the overall complication rate was reported $3.3 \%$, with the major complication rate of $1.4 \%$ [15]. Life threatening complications have not been reported with RFA.

Reported major complications, which did not resolve in order of frequency are: permanent vocal cord palsy with inspiratory stridor and without dysphonia [10,16,19,30], hypothyroidism [15] and brachial plexus injury [19]. There are case reports about third degree skin burn [42] and rupture of benign thyroid nodules [15,43].

Minor complications included hematoma, vomiting, skin burn, fever, edema and pain only if persisting for more than 2-3 days [9]. Hematomas can be controlled by neck compression or ice application [21] and usually they disappear within 1 or 2 weeks [15].

The most common side effect was various degree pain and discomfort at the time of procedure. If the patient cannot tolerate pain, it is recommended to reduce the generator output [12] or to use local ice application [15]. There was no patient to whose treatment was not completed due to pain.

Bipolar RFA has some advantages compared with monopolar there is no need for grounding pads, therefore burns at the grounding pad site can be avoided. Bipolar RFA reduces risk of RFA associated malfunction of implanted electrical devices, especially for elderly patients [28].

To reduce the risk of complications it is necessary for the operator to strictly trace the electrode tip during the procedure, to have a thorough knowledge of the neck anatomy and to be experienced in image-guided interventions [12].

Procedure complications do not differ between malignant and benign nodules although there are differences in the procedure and affected neck anatomy. The only permanent complication reported was vocal cord paralysis in four cases [31,32,34,36].

In summary, RF ablation for recurrent thyroid cancers show lower morbidity and mortality and lower cost, as well as better suitability for real - time guidance of the treatment procedure. In cases of overt vascular encasement or massive calcification that can cause difficulties in terms of insufficient ablation or incomplete approach, clinical effectiveness of outpatient treatment and management form of RFA is unclear [33].

\section{Post-procedural evaluation criteria}

\subsection{Volume reduction}

The most important indicator for RFA efficacy is thyroid nodule volume reduction after treatment. Reported mean data varies from 50.7 to $93.5 \%$ of volume reduction [see benign nodule table]. Volume reduction ratio (VRR) for the most of malignant nodule studies are more than $90 \%$, except for two $[33,38]$. Rarely nodules disappear completely, mostly there are scar tissue left, appearing predominantly hypoechoic or has hyperechoic areas in the center of the treated area [44]. Follow up period can affect study results about volume reduction [44]. As thyroid nodule shrinkage occurs gradually - mostly rapidly at first month and further continues until at least 6 months [24], the studies with longer follow-up mostly are needed to see long-term results and risk of recurrence.

Better response at first for mixed or mainly cystic nodules might be due to the homogeneous conduction of heat and the absence of a heat sink effect [30] as well as fluid aspiration before procedure.

Mainly solid tumors (solid portion $>30 \%$ ) decrease in volume according to the mean value if the tumor is completely ablated. 


\subsection{Nodule functionality}

Results show that nodule functionality does not change ablation results and complication risk. One of studies even showed that hot nodules show a slightly but not significantly greater response than cold nodules [24]. Also, it is important to add that all studies with included symptomatic "hot" nodules in scintigraphy showed complete recovery of hyperthyroidism in $23-40 \%$ of cases or partial remission of thyroid hyperfunction by 40-79\% [24-26]. Multicenter study demonstrated that hyperthyroidism improved (in $100 \%$ ) or completely normalized (in $81.8 \%$ ) in the study of 44 patients without development of hypothyroidism [9].

\subsection{Clinical symptom reduction}

One of most important post procedural evaluation criteria is change in patient's clinical problems - pressure symptoms and cosmetic issues. As these, are very subjective criteria, studies used $10 \mathrm{~cm}$ visual scale to rate pressure symptoms and physicians recorded a cosmetic grade to evaluate nodule mass. An improvement of symptoms and cosmetic problems was demonstrated in all studies [see benign nodule table].

\subsection{Efficacy}

The most important criteria of RFA effectiveness is volume reduction ratio. Other criteria that reveal efficacy of this method is therapeutic success rate (recurrence rate), complete disappearance of the treated tumor and decreased serum $\mathrm{Tg}$ (thyroglobulin) concentration [see malignant nodule table] - for athyreotic patients, $\mathrm{Tg}$ is expected to be undetectable, whereas detectable Tg levels are associated with recurrent or persistent disease [45].

Efficacy of bipolar system is comparable with monopolar RFA technique. Reported results are volume reduction ratios of $>50 \%$ and improvement of compressive and cosmetic symptoms [23]. Usage of bipolar RFA on symptomatic thyroid nodules have shown a median increase in Thyroglobulin ( $\mathrm{Tg}$ ) level and normalization of scintigraphy findings [28] therefore this system has potential to be used as a radiation - free alternative to radioiodine therapy. However, further studies about bipolar RFA with a larger study population with longer follow - up period are necessary.

\section{Follow-up}

\subsection{Benign nodules}

Generally, ultrasound follow-up examination is carried out at the 1-, 3- and 6-month visits and after that one time in every 6-12 month, but it varies among studies from 1 to 92 months, on average 16.5 months [see benign nodule table].

The ultrasound findings, laboratory data and clinical problems are evaluated during the follow-up period. For functioning nodules also scintigraphy examination can be advised to evaluate changes [9].

Main features in need to be assessed in ultrasonography are: changes in nodule size, echogenicity and vascularity. Ablation areas are hypoechoic and tend to reduce in sizes. Presence of intra-nodal vascularity after RFA is important to indicate the necessity of repeated RFA procedure [36] because it should disappear in fully ablated regions.

Follow up period can be discontinued if treated nodule disappears completely or remains as a small scarring tissue [44].

\subsection{Malignant nodules}

Follow up period for recurrent thyroid carcinoma nodules should be longer and more active. Two main criteria are mandatory to evaluate ablated tissue vascularity and serum thyroglobulin ( $\mathrm{Tg}$ ) level. Vascularity may be assessed by imaging: computed tomography [46], magnetic resonance imaging within staging protocol [47] and Color Doppler ultrasound - loss of color signal within a treated lesion that was previously hypervascular is adequate evidence of appropriate thermal coagulation [31] and any part of nodule that maintains vascularity needs an additional ablation session or different therapy. Invariability or increase of serum thyroglobulin ( $\mathrm{Tg}$ ) concentration can identify patients with residual tumor [45]. There was only one study with available data about increased Tg levels for three patients [33] and authors considered that it is related to tumor biological behaviors, for other studies $\mathrm{Tg}$ level decreased or was even below the detectable level [malignant nodule table].

\section{Discussion}

Thyroid nodules and nodular goiter is a common clinical problem with a long-term established solution - surgery. However, in the past two decades various minimally invasive nonsurgical techniques have been proposed to avoid major traumatizing of the skin and tissues of the neck, general anesthesia and to improve the quality of life for various populations.

Regardless to supposition that RFA is safe, the Korean Society of Thyroid Radiology advise to take a special care in cases of contralateral vocal cord palsy, pregnant women and patients with serious health issues [12] as well as patients with cardiac pacemakers or implantable cardioverter-defibrillators to avoid unnecessary risk. It has been recommended to do RFA in brief and intermittent bursts at the lowest energy levels, to maximize the distance between electrode and cardiac device, to monitor pulse during the procedure and to perform ECG evaluation after treatment [14].

In addition to RFA, there are also available other minimally invasive treatment methods e.g. percutaneous ethanol injection (PEI), laser ablation (LA), microwave ablation (MWA) and high intensity focused ultrasound (HIFU). It should be noted that RFA is the only minimally invasive method that has been increasingly used for recurrent thyroid cancer.

Percutaneous ethanol injection (PEI) is the oldest minimally invasive technique for thyroid nodule treatment [14] since it is a simple, rapid and relatively inexpensive procedure [13]. PEI efficacy is mainly affected by proportion of solid and cystic component since it is more effective in the treatment of cystic nodules (cystic component >90\%) [48]. Reported volume reduction has been observed in $82.4-96.9 \%$ cases of cystic nodules and $65.8-86.2 \%$ in predominantly cystic nodules. It can be explained by the fact that solid component is thought to be more resistant to ethanol and increased vascularity of nodule is increasing the drainage of ethanol [49]. Studies have reported that PEI's efficacy decreases as solid component increase and this method is less effective in the treatment of nodules with $>20 \%$ solid component [50]. Therefore, in patients with incompletely resolved clinical problems, the RFA may be advised as additional treatment modality [51]. Comparing the two methods regarding cystic nodules, PEI is superior in terms of the number of treatment sessions required [49,52], presents shorter procedure time [53] and less periprocedural pain than with RFA [53]. Nevertheless, the best treatment modality for predominantly cystic thyroid nodules is still under debate because recurrence rate after PEI is reported 26-38.3\% [54], therefore a combination of both methods may be advised for benign thyroid nodules [51]. 
Laser ablation (LA) can be used for the treatment of cold, solid, benign thyroid nodules. Reported volume reduction ranges from 48.8 to $84 \%[13,55,56]$. LA most important advantage is that the thin and flexible laser fibers render this procedure as precise by inducing a well - defined area of ablation $[44,55]$. Also important beneficial factors are: low cost, easy to control and provides results stable for at least three years after procedure [55,57]. There are controversial data about how nodule structure, morphology and size affects treatment effectiveness depending on the heterogeneity of the treatment procedures used [56]. To establish selection criteria and determine efficacy, prospective randomized studies on LA versus RFA must be conducted, although review articles admit that the efficacy of RFA appears to be slightly superior and with less adverse effects [44,57]. However, it worth of note that in both treatment modalities major complications are rare [13].

MWA and HIFU are still under investigation since have been only recently introduced. HIFU uses a focused high energy ultrasound beam for thermal tissue ablation in targeted zone with minimal effect on the surrounding tissue [58]. It's main advantages are non-invasiveness and few adverse effects. This advantage is also the source of HIFU's major limitation - technical accessibility [58]. Patient BMI, nodule localization (maximal depth of $2.8 \mathrm{~cm}$ ) [27] and long procedural duration needs to be considered when decision is made in favor of HIFU. In addition, the assessment of nodule vascularization and the initial echogenicity of the nodule seem to be important, as predictors of the efficacy of the procedure. Reported volume reduction is less than $50 \%$ [27,58], but follow up period in these studies have been relatively short and included small group of patients.

MWA contrary to HIFU can be applicable to miscellaneous thyroid nodules - cystic, mixed and solid. Nodules with cystic component show better response due to homogeneous conduction of heat and the absence of heat sink effect [59]. There have not been reported any major complications yet and further studies are necessary to judge the safety of this method. The efficacy of both methods has not been established up to this date, therefore comparison would not be incontrovertible. In terms of procedure accessibility, cost and duration - the RFA procedure is superior.

RFA does not overlap with the conventional and established treatment methods - surgery and radioiodine therapy for thyroid nodules [14]. It is mandatory to evaluate every patient individually as for inclusion criteria and for follow-up as proved for liver tumor characterization and for liver tumor RFA treatment assessment [21,60-63].

CEUS, US-elastography and virtual navigation may offer further prospects from planning the treatment and assess the subsequent results by multi-parametric approach.

\subsection{Limitations of the review}

We have to mention that this review presents some drawbacks, such as: first, heterogeneity of study designs and result reports lead to difficult comparison and evaluation of the studies. Second, inclusion criteria are strict and include only limited cases, consequently most of analyzed studies had small population or insufficient statistical reliability. Third, in the studies about benign thyroid nodules there is absence of histological confirmation of nodule structure, although some of the centers recently show trend to perform core needle biopsy prior RFA procedure [64,65] as well as genetic assessment of differentiated thyroid cancer [66].

\section{Conclusions}

Based on the existing literature, nonsurgical, minimally invasive techniques for the management of thyroid nodules are developing rapidly and are tended to the patient - centered/personalized approach. RFA have been proved to be effective and safe in cases of benign thyroid nodules and promising alternative treatment method to surgery for patients with recurrent papillary thyroid cancer.

However, future multicenter studies are warranted to better define multi-parametric patient selection criteria and clarify the role, efficacy, advantages and limitations of the RFA technique in the variety of thyroid nodular disease.

\section{Sources of funding}

None.

\section{Author contribution}

Maija Radzina, Vito Cantisani, Madara Rauda, Michael Bachmann Nielsen, Caroline Ewertsen, Ferdinando D'Ambrosio, Peteris Prieditis and Salvatore Sorrenti contributed to define the content, methodological strategy and the aims of the review. They all contributed to write the first draft of the manuscript and all agree to its final version.

\section{Conflicts of interest}

The authors declare no conflict of interest.

\section{Guarantor}

Salvatore Sorrenti.

\section{References}

[1] L.R. Remonti, et al., Thyroid ultrasound features and risk of carcinoma: a systematic review and meta-analysis of observational studies, Thyroid 25 (5) (2015) 538-550.

[2] A.E. Brander, et al., Importance of thyroid abnormalities detected at US screening: a 5-year follow-up, Radiology 215 (3) (2000) 801-806.

[3] M.C. Chammas, et al., Thyroid nodules: evaluation with power Doppler and duplex Doppler ultrasound, Otolaryngol. Head Neck Surg. 132 (6) (2005) $874-882$.

[4] J. Tramalloni, et al., Imaging of thyroid nodules, J. Radiol. 80 (3) (1999) $271-277$.

[5] C.C. Reading, et al., Sonography of thyroid nodules: a "classic pattern" diagnostic approach, Ultrasound Q. 21 (3) (2005) 157-165.

[6] B.R. Haugen, et al., 2015 American thyroid association management guidelines for adult patients with thyroid nodules and differentiated thyroid cancer: the American thyroid association guidelines task force on thyroid nodules and differentiated thyroid cancer, Thyroid 26 (1) (2016) 1-133.

[7] L. Davies, H.G. Welch, Increasing incidence of thyroid cancer in the United States, 1973-2002, JAMA 295 (18) (2006) 2164-2167.

[8] D.A. Meier, M.M. Kaplan, Radioiodine uptake and thyroid scintiscanning, Endocrinol. Metab. Clin. North Am. 30 (2) (2001) 291-313 (viii).

[9] J.Y. Sung, et al., Radiofrequency ablation for autonomously functioning thyroid nodules: a multicenter study, Thyroid 25 (1) (2015) 112-117.

[10] Y.S. Kim, et al., Radiofrequency ablation of benign cold thyroid nodules: initial clinical experience, Thyroid 16 (4) (2006) 361-367.

[11] R. Garberoglio, et al., Radiofrequency ablation for thyroid nodules: which indications? The first Italian opinion statement, J. Ultrasound 18 (4) (2015) 423-430.

[12] D.G. Na, et al., Radiofrequency ablation of benign thyroid nodules and recurrent thyroid cancers: consensus statement and recommendations, Korean J. Radiol. 13 (2) (2012) 117-125.

[13] E. Papini, et al., The advent of ultrasound-guided ablation techniques in nodular thyroid disease: towards a patient-tailored approach, Best. Pract. Res. Clin. Endocrinol. Metab. 28 (4) (2014) 601-618.

[14] S. Bernardi, et al., Radiofrequency ablation for benign thyroid nodules, J. Endocrinol. Investig. 39 (9) (2016) 1003-1013.

[15] J.H. Baek, et al., Complications encountered in the treatment of benign thyroid nodules with US-guided radiofrequency ablation: a multicenter study, Radiology 262 (1) (2012) 335-342.

[16] R. Cesareo, et al., Prospective study of effectiveness of ultrasound-guided radiofrequency ablation versus control group in patients affected by benign thyroid nodules, J. Clin. Endocrinol. Metab. 100 (2) (2015) 460-466.

[17] M. Deandrea, et al., Efficacy and safety of radiofrequency ablation versus 
observation for nonfunctioning benign thyroid nodules: a randomized controlled international collaborative trial, Thyroid 25 (8) (2015) 890-896.

[18] J.H. Baek, et al., Benign predominantly solid thyroid nodules: prospective study of efficacy of sonographically guided radiofrequency ablation versus control condition, AJR Am. J. Roentgenol. 194 (4) (2010) 1137-1142.

[19] H.K. Lim, et al., Radiofrequency ablation of benign non-functioning thyroid nodules: 4-year follow-up results for 111 patients, Eur. Radiol. 23 (4) (2013) 1044-1049.

[20] E.J. Ha, et al., Radiofrequency ablation of benign thyroid nodules does not affect thyroid function in patients with previous lobectomy, Thyroid 23 (3) (2013) 289-293.

[21] G. Turtulici, et al., Percutaneous radiofrequency ablation of benign thyroid nodules assisted by a virtual needle tracking system, Ultrasound Med. Biol. 40 (7) (2014) 1447-1452.

[22] E. Aysan, et al., Single-session radiofrequency ablation on benign thyroid nodules: a prospective single center study : radiofrequency ablation on thyroid, Langenbecks Arch. Surg. 401 (3) (2016) 357-363.

[23] X.L. Li, et al., Treatment efficacy and safety of ultrasound-guided percutaneous bipolar radiofrequency ablation for benign thyroid nodules, Br. J. Radiol. 89 (1059) (2016) 20150858.

[24] M. Deandrea, et al., US-guided percutaneous radiofrequency thermal ablation for the treatment of solid benign hyperfunctioning or compressive thyroid nodules, Ultrasound Med. Biol. 34 (5) (2008) 784-791.

[25] S. Spiezia, et al., Thyroid nodules and related symptoms are stably controlled two years after radiofrequency thermal ablation, Thyroid 19 (3) (2009) 219-225.

[26] A. Faggiano, et al., Thyroid nodules treated with percutaneous radiofrequency thermal ablation: a comparative study, J. Clin. Endocrinol. Metab. 97 (12) (2012) 4439-4445.

[27] H. Korkusuz, et al., Volume reduction of benign thyroid nodules 3 months after a single treatment with high-intensity focused ultrasound (HIFU), J. Ther. Ultrasound 3 (2015) 4.

[28] Y. Korkusuz, et al., Bipolar radiofrequency ablation of benign symptomatic thyroid nodules: initial experience, Rofo 188 (7) (2016) 671-675.

[29] K.D. Kohlhase, et al., Bipolar radiofrequency ablation of benign thyroid nodules using a multiple overlapping shot technique in a 3-month follow-up, Int. J. Hyperth. 32 (5) (2016) 511-516.

[30] W.K. Jeong, et al., Radiofrequency ablation of benign thyroid nodules: safety and imaging follow-up in 236 patients, Eur. Radiol. 18 (6) (2008) 1244-1250.

[31] J.M. Monchik, et al., Radiofrequency ablation and percutaneous ethanol injection treatment for recurrent local and distant well-differentiated thyroid carcinoma, Ann. Surg. 244 (2) (2006) 296-304.

[32] S.J. Lee, et al., Radiofrequency ablation to treat loco-regional recurrence of well-differentiated thyroid carcinoma, Korean J. Radiol. 15 (6) (2014) $817-826$.

[33] K.W. Park, et al., Inoperable symptomatic recurrent thyroid cancers: preliminary result of radiofrequency ablation, Ann. Surg. Oncol. 18 (9) (2011) 2564-2568.

[34] J.P. Guenette, J.M. Monchik, D.E. Dupuy, Image-guided ablation of postsurgical locoregional recurrence of biopsy-proven well-differentiated thyroid carcinoma, J. Vasc. Interv. Radiol. 24 (5) (2013) 672-679.

[35] M. Zhang, et al., Efficacy and safety of ultrasound-guided radiofrequency ablation for treating low-risk papillary thyroid microcarcinoma: a prospective study, Thyroid 26 (11) (2016) 1581-1587.

[36] J.H. Baek, et al., Locoregional control of metastatic well-differentiated thyroid cancer by ultrasound-guided radiofrequency ablation, AJR Am. J. Roentgenol. 197 (2) (2011) W331-W336.

[37] H.K. Lim, et al., Efficacy and safety of radiofrequency ablation for treating locoregional recurrence from papillary thyroid cancer, Eur. Radiol. 25 (1) (2015) 163-170.

[38] J.H. Kim, et al., Efficacy and safety of radiofrequency ablation for treatment of locally recurrent thyroid cancers smaller than 2 cm, Radiology 276 (3) (2015) 909-918.

[39] J.H. Shin, et al., Radiofrequency ablation of thyroid nodules: basic principles and clinical application, Int. J. Endocrinol. 2012 (2012) 919650.

[40] H.Y. Kim, et al., Primary papillary thyroid carcinoma previously treated incompletely with radiofrequency ablation, J. Cancer Res. Ther. 6 (3) (2010) $310-312$.

[41] C. Dobrinja, et al., Surgical and pathological changes after radiofrequency ablation of thyroid nodules, Int. J. Endocrinol. 2015 (2015) 576576.

[42] S. Bernardi, et al., Full-thickness skin burn caused by radiofrequency ablation of a benign thyroid nodule, Thyroid 26 (1) (2016) 183-184.

[43] J.H. Shin, et al., Rupture of benign thyroid tumors after radio-frequency ablation, AJNR Am. J. Neuroradiol. 32 (11) (2011) 2165-2169.

[44] J.H. Baek, et al., Thermal ablation for benign thyroid nodules: radiofrequency and laser, Korean J. Radiol. 12 (5) (2011) 525-540.

[45] L. Giovanella, et al., Unstimulated highly sensitive thyroglobulin in follow-up of differentiated thyroid cancer patients: a meta-analysis, J. Clin. Endocrinol. Metab. 99 (2) (2014) 440-447.

[46] A.L. Mitchell, et al., Management of thyroid cancer: United Kingdom national multidisciplinary guidelines, J. Laryngol. Otol. 130 (S2) (2016) S150-S160.

[47] R. Warren Frunzac, M. Richards, Computed tomography and magnetic resonance imaging of the thyroid and parathyroid glands, Front. Horm. Res. 45 (2016) 16-23.

[48] Y.J. Kim, et al., Cystic versus predominantly cystic thyroid nodules: efficacy of ethanol ablation and analysis of related factors, Eur. Radiol. 22 (7) (2012) 1573-1578.

[49] J.Y. Sung, et al., Single-session treatment of benign cystic thyroid nodules with ethanol versus radiofrequency ablation: a prospective randomized study, Radiology 269 (1) (2013) 293-300.

[50] S.W. Jang, et al., How to manage the patients with unsatisfactory results after ethanol ablation for thyroid nodules: role of radiofrequency ablation, Eur. J. Radiol. 81 (5) (2012) 905-910.

[51] J.H. Lee, et al., Radiofrequency ablation (RFA) of benign thyroid nodules in patients with incompletely resolved clinical problems after ethanol ablation (EA), World J. Surg. 34 (7) (2010) 1488-1493.

[52] J.Y. Sung, et al., Optimum first-line treatment technique for benign cystic thyroid nodules: ethanol ablation or radiofrequency ablation? AJR Am. J. Roentgenol. 196 (2) (2011) W210-W214.

[53] J.H. Baek, et al., Radiofrequency versus ethanol ablation for treating predominantly cystic thyroid nodules: a randomized clinical trial, Korean J. Radiol. 16 (6) (2015) 1332-1340.

[54] C.H. Suh, et al., Ethanol ablation of predominantly cystic thyroid nodules: evaluation of recurrence rate and factors related to recurrence, Clin. Radiol. 70 (1) (2015) 42-47.

[55] H. Dossing, F.N. Bennedbaek, L. Hegedus, Long-term outcome following interstitial laser photocoagulation of benign cold thyroid nodules, Eur. J. Endocrinol. 165 (1) (2011) 123-128.

[56] G. Achille, et al., Ultrasound-guided percutaneous laser ablation in treating symptomatic solid benign thyroid nodules: our experience in 45 patients, Head Neck 38 (5) (2016) 677-682.

[57] E. Papini, et al., Long-term efficacy of ultrasound-guided laser ablation for benign solid thyroid nodules. Results of a three-year multicenter prospective randomized trial, J. Clin. Endocrinol. Metab. 99 (10) (2014) 3653-3659.

[58] R.D. Kovatcheva, et al., Benign solid thyroid nodules: US-guided high-intensity focused ultrasound ablation-initial clinical outcomes, Radiology 276 (2) (2015) 597-605.

[59] W. Yue, et al., Ultrasound guided percutaneous microwave ablation of benign thyroid nodules: safety and imaging follow-up in 222 patients, Eur. J. Radiol. 82 (1) (2013) e11-6.

[60] M.F. Meloni, et al., Contrast enhanced ultrasound in the evaluation and percutaneous treatment of hepatic and renal tumors, Eur. J. Radiol. 84 (9) (2015) 1666-1674.

[61] V. Cantisani, et al., Ultrasound elastography in the evaluation of thyroid pathology. Current status, Eur. J. Radiol. 83 (3) (2014) 420-428.

[62] M. D'Onofrio, et al., Intrahepatic peripheral cholangiocarcinoma (IPCC): comparison between perfusion ultrasound and CT imaging, Radiol. Med. 113 (1) (2008) 76-86.

[63] C.F. Dietrich, et al., EFSUMB guidelines on interventional ultrasound (INVUS), Part III - abdominal treatment procedures (Short Version), Ultraschall Med. 37 (1) (2016) 27-45.

[64] D.G. Na, et al., Core-needle biopsy is more useful than repeat fine-needle aspiration in thyroid nodules read as nondiagnostic or atypia of undetermined significance by the Bethesda system for reporting thyroid cytopathology, Thyroid 22 (5) (2012) 468-475.

[65] E.J. Ha, et al., Sonographically suspicious thyroid nodules with initially benign cytologic results: the role of a core needle biopsy, Thyroid 23 (6) (2013) 703-708.

[66] V. Marotta, et al., Germline polymorphisms of the VEGF-pathway predict recurrence in non-advanced differentiated thyroid cancer, J. Clin. Endocrinol. Metab. (2016 Nov 16), http://dx.doi.org/10.1210/jc.2016-2555. 